УДК 624.1

\author{
И.Н. Чиж, Г.М. Скибин
}

Южно-Российский государственный технический университет (Новочеркасский политехнический институт) им. М.И. Платова, Новочеркасск, Россия

\title{
РАЗРАБОТКА МЕТОДИКИ РАСЧЕТА ОПТИМАЛЬНОЙ
}

\section{ГЛУБИНЫ ЗАЛОЖЕНИЯ РАЗДЕЛИТЕЛЬНОГО ШПУНТОВОГО РЯДА В СТЕСНЕННЫХ УСЛОВИЯХ СТРОИТЕЛЬСТВА}

В результате экспериментально-численного исследования выявлены закономерности влияния шпунтового ограждения на изменение напряженно-деформированного состояния основания исторически сложившейся застройки, расположенной в зоне влияния нового строительства и представленной различными типами зданий и категорией их технического состояния. Выявленные закономерности заложены в разработанную методику расчета оптимальной глубины заложения разделительной стенки, реализованную в компьютерной программе «Расчет оптимальной глубины заложения разделительной стенки».

Ключевые слова: существующие строения, возводимое здание, фундамент, шпунтовое ограждение, влияние, эксперимент, расчет, результаты, оптимальный, глубина заложения.

\author{
I.N. Chizh, G.M. Skibin
}

South-Russian State Technical University (Novocherkassk Polytechnic Institute) named. M.I. Platov, Novocherkassk, Russian Federation

\section{DEVELOPMENT OF METHOD OF CALCULATION OF THE SHEETING PILE'S OPTIMAL DEPTH IN THE CONSTRAINED CONDITIONS OF CONSTRUCTION}

In the result of experimental and numerical study finded regularities of influence sheeting pile on the change in the stress-strain state the grounds of the historic building, located in the zone of influence of the new construction and presents different types of buildings and their technical condition. The finded regularities inherent in the developed method of calculation of the optimum depth of the separation wall, which is implemented in a computer program "Calculation of optimal depth separation wall».

Keywords: existing buildings, erected building, foundation, sheeting pile, influence, experiment, calculation, results, optimal, depth.

В условиях современного строительства весьма актуальна проблема дефицита городских территорий. Возведение новых зданий в непосредственной близости от существующих строений влечет за со- 
бой дополнительные деформации исторически сложившейся застройки. Поэтому строительство в стесненных условиях обязательно должно сопровождаться специальными мероприятиями по обеспечению сохранности существующих строений, учитывающих состояние их строительных конструкций. Одним из таких мероприятий является устройство ограждающих конструкций (забирка, шпунт, стены в грунте различных конструкций и способов их изготовления), популярность которых значительно возросла за последние годы.

На основе анализа нормативной литературы авторами составлены отношения дополнительных осадок к основным $\left(S_{a d, u}^{\max } / S_{u}^{\max }\right)$, полученным до влияния нового строительства, существующим строениям различных типов и категорий технического состояния (табл. 1).

Таким образом, полученные отношения $S_{a d, u}^{\max } / S_{u}^{\max }$ для каждого случая определили граничные условия исследований, т.е. глубина заложения шпунтового ряда считалась оптимальной, если выполнялось условие из табл. 1.

Таблица 1

Предельные дополнительные деформации существующих строений, см / $S_{a d, u}^{\max } / S_{u}^{\max }$

\begin{tabular}{|l|c|c|c|c|}
\hline \multicolumn{1}{|c|}{ Здания } & \multirow{2}{*}{$\begin{array}{l}\text { З } \\
\text { max }\end{array}$} & \multicolumn{2}{|c|}{$\begin{array}{c}\text { Категория техническо- } \\
\text { го состояния зданий }\end{array}$} \\
\cline { 3 - 5 } & & I & II & III \\
\hline $\begin{array}{l}\text { Гражданские и производственные одноэтаж- } \\
\text { ные и многоэтажные здания с полным железо- } \\
\text { бетонным каркасом }\end{array}$ & 10 & $\frac{5}{0,5}$ & $\frac{3}{0,3}$ & $\frac{2}{0,2}$ \\
\hline $\begin{array}{l}\text { Многоэтажные бескаркасные здания с несу- } \\
\text { щими стенами из крупных панелей }\end{array}$ & 12 & $\frac{4}{0,33}$ & $\frac{3}{0,25}$ & $\frac{2}{0,17}$ \\
\hline $\begin{array}{l}\text { Многоэтажные бескаркасные здания с несу- } \\
\text { щими стенами их крупных блоков или кир- } \\
\text { пичной кладки без армирования }\end{array}$ & 12 & $\frac{4}{0,33}$ & $\frac{3}{0,25}$ & $\frac{1}{0,08}$ \\
\hline $\begin{array}{l}\text { Многоэтажные бескаркасные здания с несущи- } \\
\text { ми стенами из кирпича или бетонных блоков с } \\
\text { арматурными или железобетонными поясами }\end{array}$ & 18 & $\frac{5}{0,28}$ & $\frac{3}{0,17}$ & $\frac{2}{0,11}$ \\
\hline $\begin{array}{l}\text { Многоэтажные и одноэтажные здания истори- } \\
\text { ческой застройки или памятники истории, ар- } \\
\text { хитектуры и культуры с несущими стенами из } \\
\text { кирпичной кладки без армирования }\end{array}$ & 12 & - & $\frac{1}{0,08}$ & $\frac{0,5}{0,04}$ \\
\hline
\end{tabular}


Модели ленточных фундаментов устанавливались на песчаное основание в одном уровне на различных расстояниях друг от друга [1], и в каждом случае подбор оптимальной глубины заложения шпунта производился в пяти его положениях между фундаментами (рис. 1).

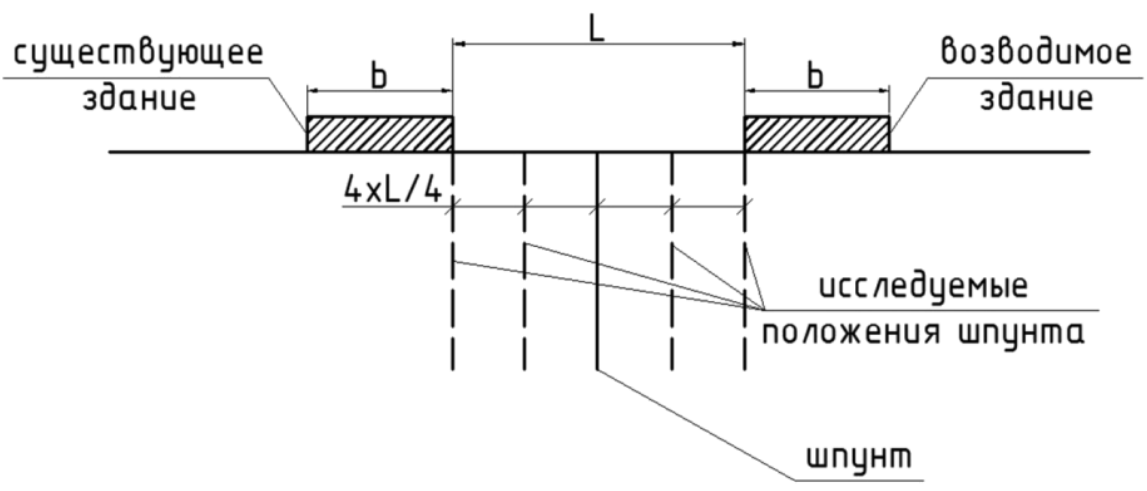

Рис. 1. Постановка исследования

По результатам экспериментов выполнена численная проверка подобранных параметров в программной комплексе PLAXIS (рис. 2), которая показала хорошую сходимость (рис. 3) [2].

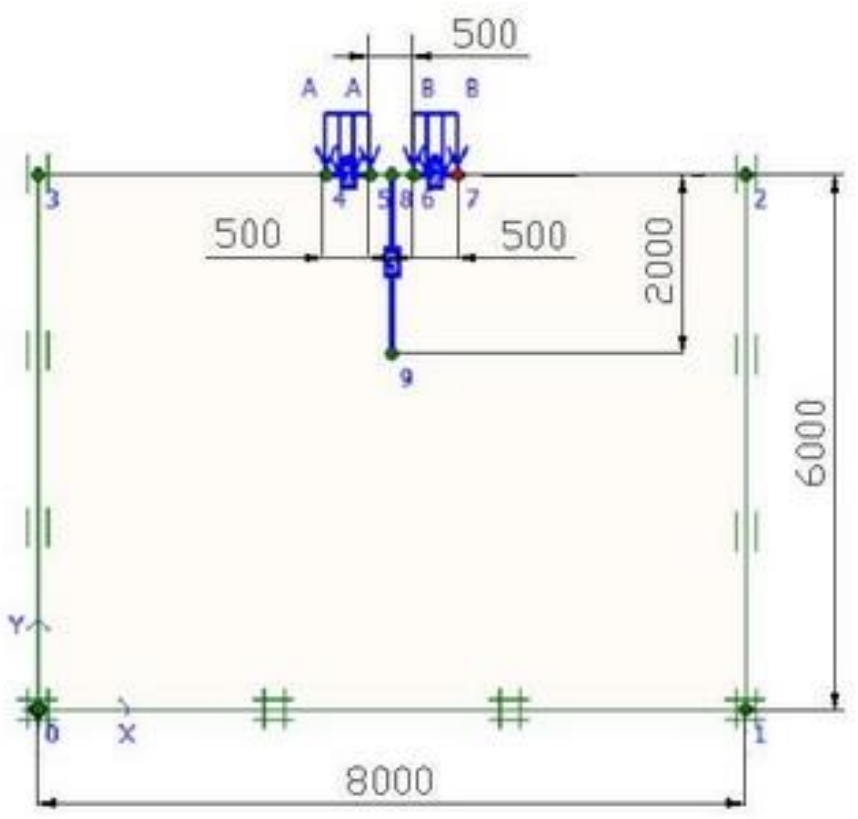

Рис. 2. Геометрическая модель численного моделирования (размеры в мм) 


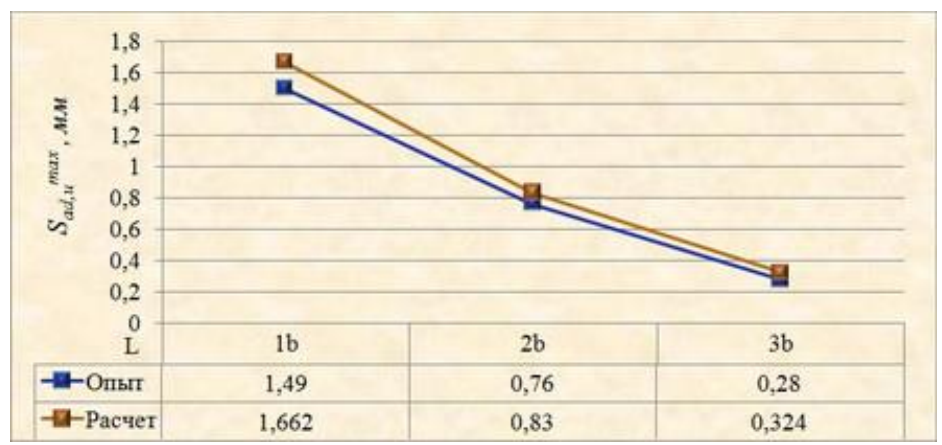

Рис. 3. График зависимости дополнительной осадки существующего здания от расстояния, на которое оно удалено от строящегося объекта

Оценка напряженно-деформированного состояния основания под соседними фундаментами выполнена в испытательной установке МФ-1 на кафедре «Промышленное, гражданское строительство, геотехника и фундаментостроение» Южно-Российского государственного политехнического университета (Новочеркасского политехнического института) им. М.И. Платова с применением тензометрических датчиков (рис. 4).

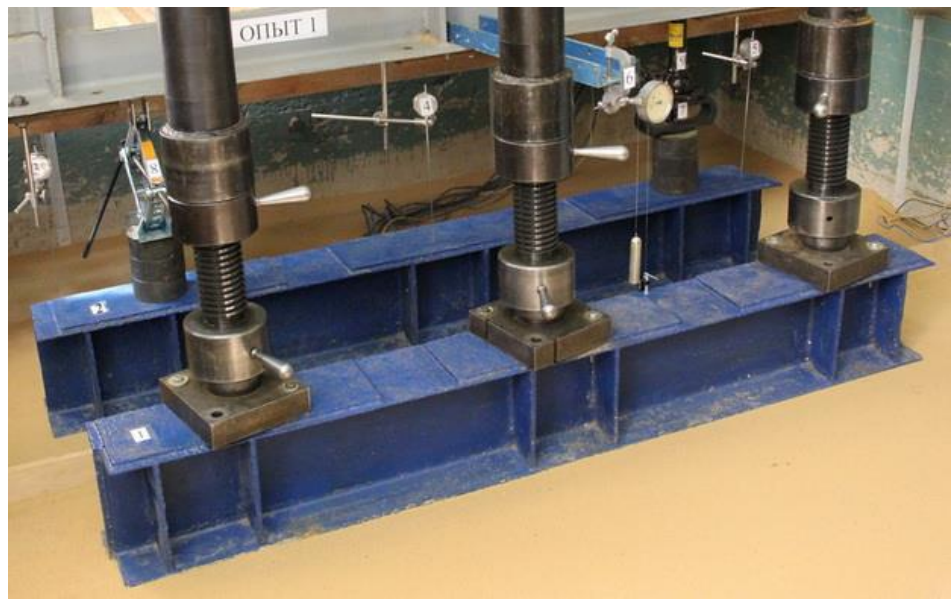

Рис. 4. Расположение измерительных приборов, устройств и моделей в МФ-1

Исследования выполнены в трех постановках: без устройства шпунта и с устройством шпунта посередине между моделями фундаментов и у модели возводимого здания. Обработка показаний тензометрических датчиков выполнялась в программе «Система обработки 
тензометрических измерений» («СОТИ»), которая позволила объединить все элементы лаборатории и этапы эксперимента, дала возможность проведения экспериментов в реальном масштабе времени с автоматизированным анализом результатов [3].

Выполнен анализ графических данных, полученных в результате численного моделирования в программном комплексе PLAXIS и экспериментального исследования в программе СОТИ на каждой стадии нагружения (рис. 5-7).

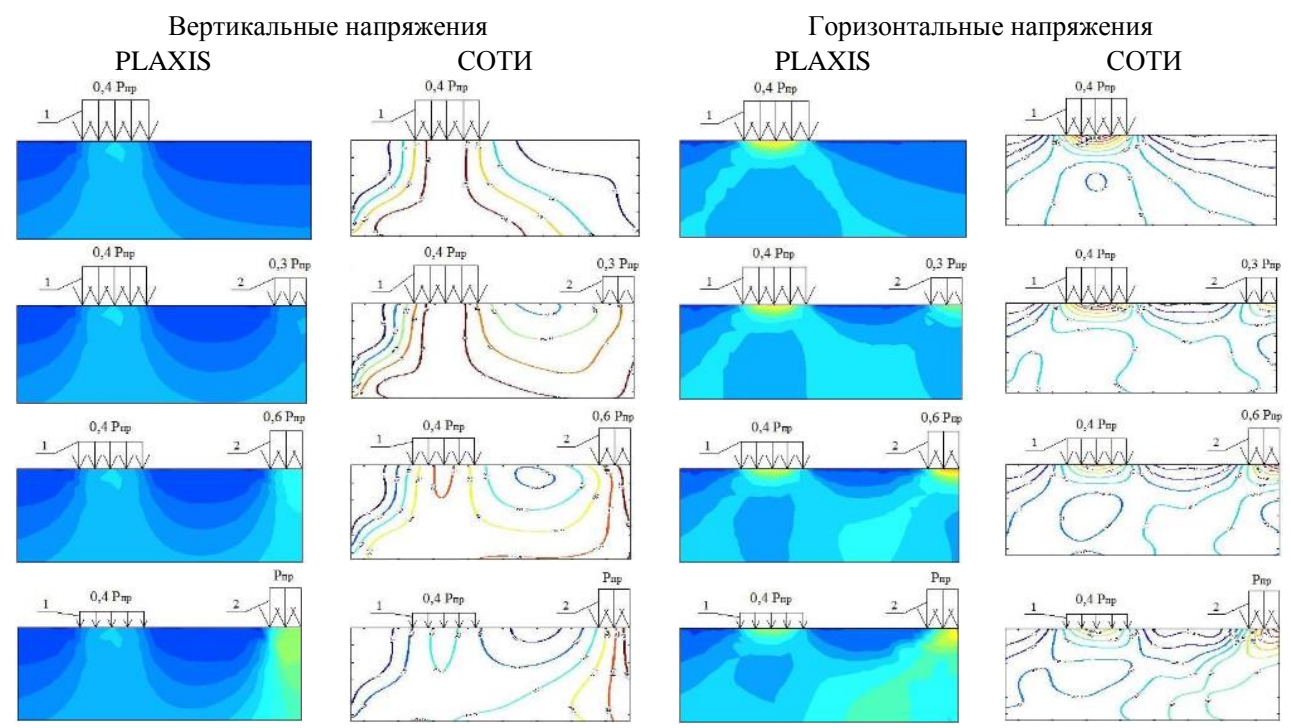

Рис. 5 Изополя и изолинии нормальных напряжений в основании под соседними фундаментами: 1 - существующий фундамент; 2 - возводимый фундамент

В основании близкорасположенных зданий зоны распределения напряжений накладываются друг на друга, формируя единое поле напряженно-деформированного состояния. Устройство разделительного ряда между соседними зданиями позволяет ограничить область распространения напряжений в грунтовом массиве, возникающих от давления строящегося объекта. Таким образом, ограждающая конструкция препятствует распределению напряжений в грунтовом основании, создаваемых возводимым зданием, на всех этапах нагружения. Установка разъединительной стенки правильной длины вплотную к фундаменту возводимого здания позволила блокировать напряжения, возникающие от нового строительства в грунтовом массиве. Характер распределения нормальных напряжений на всех стадиях нагружения остается практи- 
чески неизменным по сравнению с ситуацией, когда основание под существующим строением не испытывало дополнительного воздействия.

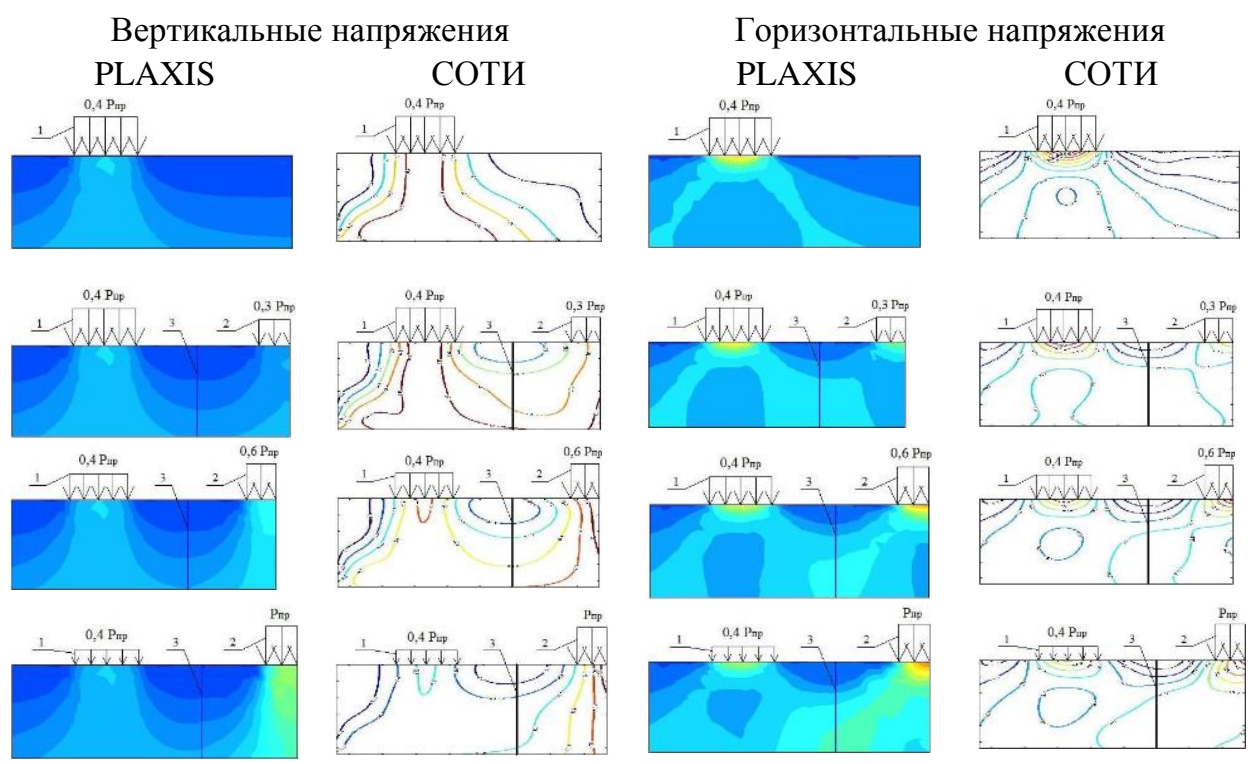

Рис. 6. Изополя и изолинии нормальных напряжений в основании под соседними фундаментами, разделенными шпунтовым рядом посередине; 1 - существующий фундамент; 2 - возводимый фундамент; 3 - шпунтовый ряд

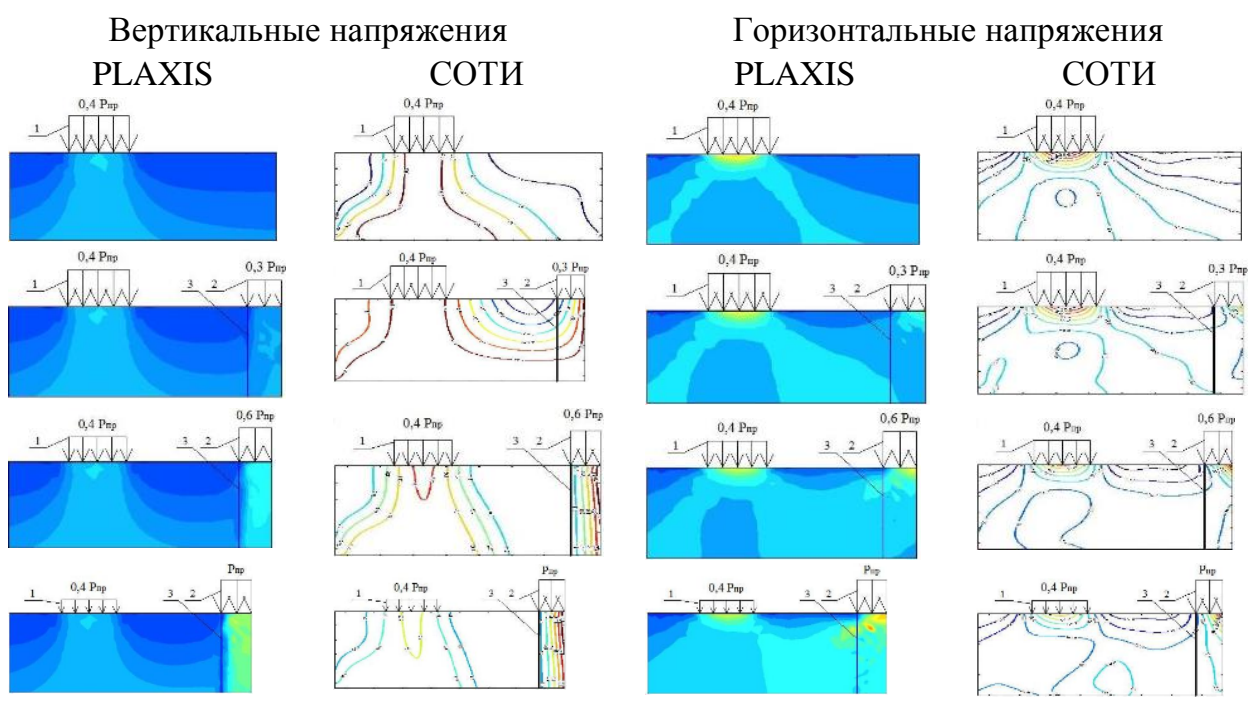

Рис. 7. Изополя и изолинии нормальных напряжений в основании под соседними фундаментами, разделенными шпунтовым рядом у возводимого фундамента: 1 - существующий фундамент; 2 - возводимый фундамент; 3 - шпунтовый ряд 
Изучение изменения напряженно-деформированного состояния песчаного основания в зоне влияния нового строительства на существующее строение на всех этапах нагружения возводимого здания позволяет сделать следующие выводы:

- устройство разделительного ряда способно значительно сократить дополнительное влияние строящегося объекта, уменьшить интенсивность изменения напряженно-деформированного состояния основания существующего фундамента на всех стадиях нагружения возводимого и обеспечить деформации основания в пределах допустимых значений;

- экспериментально доказана наибольшая эффективность ограждающей конструкции, установленной вплотную к возводимому объекту.

В результате экспериментально-численного исследования составлена таблица подбора оптимальных параметров шпунтового ограждения, выполняющего роль защитного мероприятия существующих строений (табл. 2).

Таблица 2

Оптимальная глубины заложения шпунта и прогнозируемое значение $S_{a d, u}^{\max } / S_{u}^{\max }$ для различных условий строительства

\begin{tabular}{|c|c|c|c|c|c|c|c|c|c|c|c|c|c|c|c|}
\hline \multirow{2}{*}{$L$} & \multirow{2}{*}{$l$} & \multicolumn{14}{|c|}{ Тип и категория технического состояния здания } \\
\hline & & A I & A II & A III & Б I & Б II & Б III & B I & B II & B III & $\Gamma \mathrm{I}$ & $\Gamma$ II & $\Gamma \mathrm{III}$ & Д ІІ & Д ІІІ \\
\hline \multirow{5}{*}{$0,5 b$} & 0 & $5 b$ & $6,5 b$ & $6,5 b$ & $6,5 \mathrm{~b}$ & $6,5 b$ & $7 b$ & $6,5 b$ & $6,5 b$ & $7 b$ & $6,5 b$ & $7 b$ & $7 b$ & $7 b$ & $7,5 b$ \\
\hline & $\mathrm{L} / 4$ & $5 b$ & $6,5 b$ & $6,5 b$ & $6 b$ & $6,5 b$ & $7 b$ & $6 b$ & $6,5 b$ & $7 b$ & $6,5 b$ & $7 b$ & $7 b$ & $7 b$ & $7 b$ \\
\hline & $\mathrm{L} / 2$ & $5 \mathrm{~b}$ & $6 \mathrm{~b}$ & $6,5 \mathrm{~b}$ & $6 \mathrm{~b}$ & $6,5 b$ & $7 b$ & $6 \mathrm{~b}$ & $6,5 b$ & $7 b$ & $6,5 b$ & $7 b$ & $7 b$ & $7 b$ & $7 \mathrm{~b}$ \\
\hline & $3 \mathrm{~L} / 4$ & $5 b$ & $6 \mathrm{~b}$ & $6,5 \mathrm{~b}$ & $6 \mathrm{~b}$ & $6,5 \mathrm{~b}$ & $7 \mathrm{~b}$ & $6 \mathrm{~b}$ & $6,5 \mathrm{~b}$ & $7 b$ & $6,5 b$ & $7 b$ & $7 \mathrm{~b}$ & $7 \mathrm{~b}$ & $7 b$ \\
\hline & $\mathrm{L}$ & $5 \mathrm{~b}$ & $6 \mathrm{~b}$ & $6,5 \mathrm{~b}$ & $5,5 \mathrm{~b}$ & $6,5 b$ & $7 \mathrm{~b}$ & $5,5 \mathrm{~b}$ & $6,5 \mathrm{~b}$ & $7 \mathrm{~b}$ & $6 \mathrm{~b}$ & $7 \mathrm{~b}$ & $7 \mathrm{~b}$ & $7 \mathrm{~b}$ & $7 \mathrm{~b}$ \\
\hline \multirow{5}{*}{$1 \mathrm{~b}$} & 0 & $4 b$ & $6 b$ & $6,5 b$ & $6 \mathrm{~b}$ & $6,5 b$ & $7 b$ & $6 \mathrm{~b}$ & $6,5 b$ & $7 b$ & $6,5 b$ & $7 b$ & $7 b$ & $7 b$ & $7,5 b$ \\
\hline & $\mathrm{L} / 4$ & $4 \mathrm{~b}$ & $6 \mathrm{~b}$ & $6,5 \mathrm{~b}$ & $6 \mathrm{~b}$ & $6,5 b$ & $7 \mathrm{~b}$ & $6 \mathrm{~b}$ & $6,5 \mathrm{~b}$ & $7 \mathrm{~b}$ & $6,5 \mathrm{~b}$ & $7 b$ & $7 b$ & $7 b$ & $7 b$ \\
\hline & $\mathrm{L} / 2$ & $3,5 b$ & $6 b$ & $6,5 b$ & $6 b$ & $6,5 b$ & $7 b$ & $6 b$ & $6,5 b$ & $7 b$ & $6,5 b$ & $7 b$ & $7 b$ & $7 b$ & $7 b$ \\
\hline & $3 \mathrm{~L} / 4$ & $3,5 b$ & $6 \mathrm{~b}$ & $6,5 b$ & $6 b$ & $6,5 b$ & $7 \mathrm{~b}$ & $6 b$ & $6,5 b$ & $7 b$ & $6 \mathrm{~b}$ & $7 b$ & $7 b$ & $7 b$ & $7 b$ \\
\hline & $\mathrm{L}$ & $3,5 b$ & $6 b$ & $6 \mathrm{~b}$ & $5,5 \mathrm{~b}$ & $6 \mathrm{~b}$ & $7 b$ & $5,5 \mathrm{~b}$ & $6 \mathrm{~b}$ & $7 b$ & $6 b$ & $7 b$ & $7 b$ & $7 b$ & $7 b$ \\
\hline \multirow{5}{*}{$1,5 \mathrm{~b}$} & 0 & - & $5 b$ & $6 b$ & $4 b$ & $5,5 b$ & $6,5 b$ & $4 \mathrm{~b}$ & $5,5 \mathrm{~b}$ & $7 b$ & $5 b$ & $6,5 b$ & $6,5 b$ & $7 b$ & $7,5 b$ \\
\hline & $\mathrm{L} / 4$ & - & $5 \mathrm{~b}$ & $5,5 \mathrm{~b}$ & $4 \mathrm{~b}$ & $5 \mathrm{~b}$ & $6 \mathrm{~b}$ & $4 \mathrm{~b}$ & $5 b$ & $7 b$ & $5 b$ & $6 \mathrm{~b}$ & $6,5 \mathrm{~b}$ & $7 b$ & $7 \mathrm{~b}$ \\
\hline & $\mathrm{L} / 2$ & - & $4,5 b$ & $5,5 \mathrm{~b}$ & $4 \mathrm{~b}$ & $5 b$ & $6 \mathrm{~b}$ & $4 \mathrm{~b}$ & $5 b$ & $6,5 b$ & $5 b$ & $6 b$ & $6,5 \mathrm{~b}$ & $6,5 b$ & $7 b$ \\
\hline & $3 \mathrm{~L} / 4$ & - & $3 b$ & $5,5 \mathrm{~b}$ & $3 b$ & $5 b$ & $6 \mathrm{~b}$ & $3 b$ & $5 b$ & $6,5 b$ & $5 b$ & $6 b$ & $6,5 \mathrm{~b}$ & $6,5 \mathrm{~b}$ & $7 b$ \\
\hline & $\mathrm{L}$ & - & $3 b$ & $4,5 \mathrm{~b}$ & $3 b$ & $4,5 \mathrm{~b}$ & $6 \mathrm{~b}$ & $3 b$ & $4,5 \mathrm{~b}$ & $6,5 \mathrm{~b}$ & $3,5 b$ & $6 \mathrm{~b}$ & $6,5 \mathrm{~b}$ & $6,5 \mathrm{~b}$ & $7 b$ \\
\hline \multirow{5}{*}{$2 b$} & 0 & - & $4 \mathrm{~b}$ & $5,5 \mathrm{~b}$ & $3 b$ & $5,5 b$ & $6 b$ & $3 b$ & $5,5 b$ & $6,5 b$ & $4,5 b$ & $6 \mathrm{~b}$ & $6,5 \mathrm{~b}$ & $6,5 b$ & $7,5 b$ \\
\hline & $\mathrm{L} / 4$ & - & $3,5 b$ & $5,5 \mathrm{~b}$ & $2,5 b$ & $4,5 \mathrm{~b}$ & $6 \mathrm{~b}$ & $2,5 b$ & $4,5 \mathrm{~b}$ & $6,5 b$ & $4 \mathrm{~b}$ & $6 b$ & $6,5 \mathrm{~b}$ & $6,5 b$ & $7 \mathrm{~b}$ \\
\hline & $\mathrm{L} / 2$ & - & $3 \mathrm{~b}$ & $5,5 \mathrm{~b}$ & $2,5 b$ & $4 \mathrm{~b}$ & $5,5 \mathrm{~b}$ & $2,5 b$ & $4 \mathrm{~b}$ & $6,5 b$ & $3 b$ & $5,5 \mathrm{~b}$ & $6,5 \mathrm{~b}$ & $6,5 \mathrm{~b}$ & $7 \mathrm{~b}$ \\
\hline & $3 \mathrm{~L} / 4$ & - & $2,5 b$ & $4,5 \mathrm{~b}$ & $1,5 b$ & $3,5 b$ & $5 \mathrm{~b}$ & $1,5 b$ & $3,5 b$ & $6,5 \mathrm{~b}$ & $3 b$ & $5 b$ & $6,5 \mathrm{~b}$ & $6,5 b$ & $6,5 b$ \\
\hline & $\mathrm{L}$ & - & $2,5 b$ & $3,5 \mathrm{~b}$ & $1 \mathrm{~b}$ & $2,5 \mathrm{~b}$ & $4,5 b$ & $1 \mathrm{~b}$ & $2,5 b$ & $6,5 b$ & $2,5 b$ & $4,5 \mathrm{~b}$ & $6 \mathrm{~b}$ & $6,5 \mathrm{~b}$ & $6,5 \mathrm{~b}$ \\
\hline \multirow{5}{*}{$2,5 \mathrm{~b}$} & 0 & - & - & $2,5 b$ & - & - & $3,5 b$ & - & - & $6 \mathrm{~b}$ & - & $3,5 b$ & $5,5 \mathrm{~b}$ & $6 \mathrm{~b}$ & $6,5 \mathrm{~b}$ \\
\hline & $\mathrm{L} / 4$ & - & - & $2 \mathrm{~b}$ & - & - & $3,5 b$ & - & - & $5,5 b$ & - & $3,5 b$ & $4,5 \mathrm{~b}$ & $5,5 \mathrm{~b}$ & $6 \mathrm{~b}$ \\
\hline & $\mathrm{L} / 2$ & - & - & $1 \mathrm{~b}$ & - & - & $2,5 b$ & - & - & $5 \mathrm{~b}$ & - & $2,5 b$ & $4 \mathrm{~b}$ & $5 b$ & $6 \mathrm{~b}$ \\
\hline & $3 \mathrm{~L} / 4$ & - & - & $1 \mathrm{~b}$ & - & - & $2,5 b$ & - & - & $4,5 \mathrm{~b}$ & - & $2,5 b$ & $4 \mathrm{~b}$ & $4,5 \mathrm{~b}$ & $5,5 \mathrm{~b}$ \\
\hline & $\mathrm{L}$ & - & - & $1 \mathrm{~b}$ & - & - & $1,5 \mathrm{~b}$ & - & - & $4,5 \mathrm{~b}$ & - & $1,5 \mathrm{~b}$ & $3 b$ & $4,5 \mathrm{~b}$ & $5,5 \mathrm{~b}$ \\
\hline
\end{tabular}


Окончание табл. 2

\begin{tabular}{|c|c|c|c|c|c|c|c|c|c|c|c|c|c|c|c|}
\hline \multirow{2}{*}{$L$} & \multirow{2}{*}{$l$} & \multicolumn{14}{|c|}{ Тип и категория технического состояния здания } \\
\hline & & A I & A II & A III & Б I & Б II & Б III & $\overline{B ~ I}$ & B II & B III & $\Gamma \mathrm{I}$ & $\Gamma \mathrm{II}$ & $\Gamma$ III & Д ІІ & Д ІІІ \\
\hline \multirow{5}{*}{$3 b$} & 0 & - & - & - & - & - & - & - & - & $3,5 \mathrm{~b}$ & - & - & $1,5 \mathrm{~b}$ & $3,5 \mathrm{~b}$ & $4,5 \mathrm{~b}$ \\
\hline & $\mathrm{L} / 4$ & - & - & - & - & - & - & - & - & $3 \mathrm{~b}$ & - & - & $1 \mathrm{~b}$ & $3 \mathrm{~b}$ & $4 \mathrm{~b}$ \\
\hline & $\mathrm{L} / 2$ & - & - & - & - & - & - & - & - & $2,5 \mathrm{~b}$ & - & - & $1 \mathrm{~b}$ & $2,5 \mathrm{~b}$ & $4 \mathrm{~b}$ \\
\hline & $3 \mathrm{~L} / 4$ & - & - & - & - & - & - & - & - & $1,5 \mathrm{~b}$ & - & - & $1 \mathrm{~b}$ & $1,5 b$ & $3,5 b$ \\
\hline & $\mathrm{L}$ & - & - & - & - & - & - & - & - & $1 \mathrm{~b}$ & - & - & $1 \mathrm{~b}$ & $1 \mathrm{~b}$ & $3,5 b$ \\
\hline
\end{tabular}

$L$ - расстояние между фундаментами; $l$ - положение шпунта; $b$ - ширина фундамента, Тип здания: А Гражданские и производственные одноэтажные и многоэтажные здания с полным железобетонным каркасом; Б Многоэтажные бескаркасные здания с несущими стенами из крупных панелей; В - Многоэтажные бескаркасные здания с несущими стенами их крупных блоков или кирпичной кладки без армирования; Г - Многоэтажные бескаркасные здания с несущими стенами из кирпича или бетонных блоков с арматурными или железобетонными поясами, Д - Многоэтажные и одноэтажные здания исторической застройки или памятники истории, архитектуры и культуры с несущими стенами из кирпичной кладки без армирования. Категория технического состояния здания: I - нормальное; II - удовлетворительное; III - неудовлетворительное.

Методика определения оптимальной глубины заложения шпунтового ряда для конкретных строительных условий предполагает:

1. Выбор типа существующего здания.

2. Выбор категории технического состояния существующего здания;

3. Выбор интервала, которому соответствует значение расстояния между существующим и возводимым зданиями.

4. Выбор положения разделительного ряда.

5. Определение оптимальной глубины заложения шпунта на пересечении выбранных параметров.

Таблица 3

Расстояние $(L)$ до строящегося объекта, на котором необходимо применять мероприятия по обеспечению сохранности существующих зданий

\begin{tabular}{|l|c|c|}
\hline \multicolumn{1}{|c|}{ Здания } & $\begin{array}{c}\text { Категория } \\
\text { состояния } \\
\text { зданий }\end{array}$ & $L_{\min } \leq L \leq L_{\max }$ \\
\hline $\begin{array}{l}\text { Гражданские и производственные одноэтажные и много- } \\
\text { этажные здания с полным железобетонным каркасом }\end{array}$ & I & $0,5 b \leq L \leq 1 b$ \\
\cline { 2 - 3 } & II & $0,5 b \leq L \leq 2 b$ \\
\hline Многоэтажные бескаркасные здания с несущими стенами & III & $0,5 b \leq L \leq 2,5 b$ \\
\cline { 2 - 3 } \begin{tabular}{l} 
из крупных панелей \\
\cline { 2 - 3 }
\end{tabular} & II & $0,5 b \leq L \leq 2 b$ \\
\hline Многоэтажные бескаркасные здания с несущими стенами & I & $0,5 b \leq L \leq 2 b$ \\
\cline { 2 - 3 } \begin{tabular}{l} 
их крупных блоков или кирпичной кладки без армирования \\
\cline { 2 - 3 }
\end{tabular} & II & $0,5 b \leq L \leq 2,5 b$ \\
\hline $\begin{array}{l}\text { Многоэтажные бескаркасные здания с несущими стенами } \\
\text { из кирпича или бетонных блоков с арматурными или желе- } \\
\text { зобетонными поясами }\end{array}$ & I & $0,5 b \leq L \leq 2 b$ \\
\cline { 2 - 3 } $\begin{array}{l}\text { Многоэтажные и одноэтажные здания исторической за- } \\
\text { стройки или памятники истории, архитектуры и культуры с } \\
\text { несущими стенами из кирпичной кладки без армирования }\end{array}$ & III & $0,5 b \leq L \leq 3 b \leq 2 b$ \\
\cline { 2 - 3 } & III & $0,5 b \leq L \leq 3 b$ \\
\hline
\end{tabular}


Разработанная методика реализована в компьютерной программе «Расчет оптимальной глубины заложения разделительной стенки».

Классификация существующих зданий, а также категории их технического состояния, приняты согласно СП 22.13330.2011 «Основания зданий и сооружений». Ширина фундаментов $(b)$, глубина заложения $(d)$ и расстояние между ними $(L)$ задается в метрах. Для каждого типа существующего здания и категории его технического состояния определено расстояние $\left(L_{\max }\right)$, на котором влияние нового строительства не превышает его предельно допустимые осадки (табл. 3).

Если в программе задать расстояние между фундаментами $L>L_{\max }$, расчет прекращается и на экран выводится сообщение «Применение шпунта не требуется!». Так как исследования проведены при максимальном сближении соседних фундаментов $L_{\min }=0,5 b$, ввод значения $L<0,5 b$ приведет к ошибке «Недопустимое значение расстояния между фундаментами!». Расчет глубины заложения ограждающей конструкции для каждого случая можно выполнить в пяти его положениях между фундаментами. После ввода значения $L$ и нажатия кнопки «Положение разделительной стенки» автоматически рассчитывается пять вариантов позиции разделительного ряда в зависимости от расстояния, на которое он удален от фундамента существующего здания ( $1=0$; $1=L / 4 ; 1=L / 2 ; 1=3 L / 4 ; 1=L)$. Нажатием на кнопку «Рассчитать оптимальную глубину заложения разделительной стенки» запускается расчет с выводом на экран оптимальной глубины заложения ограждающей конструкции в метрах и прогнозируемого значения $S_{a d, u}^{\max } / S_{u}^{\max }$ для заданных параметров.

Последовательность расчета оптимальной глубины заложения разделительной стенки в разработанной компьютерной программе:

1) открыть программу «Расчет оптимальной глубины заложения разделительной стенки»;

2) выбрать тип существующего здания;

3) выбрать категорию технического состояния существующего здания;

4) ввод значения расстояния между фундаментами существующего и возводимого здания;

5) ввод значения глубины заложения фундамента возводимого здания;

6) ввод значения ширины фундамента возводимого здания (рис. 8); 


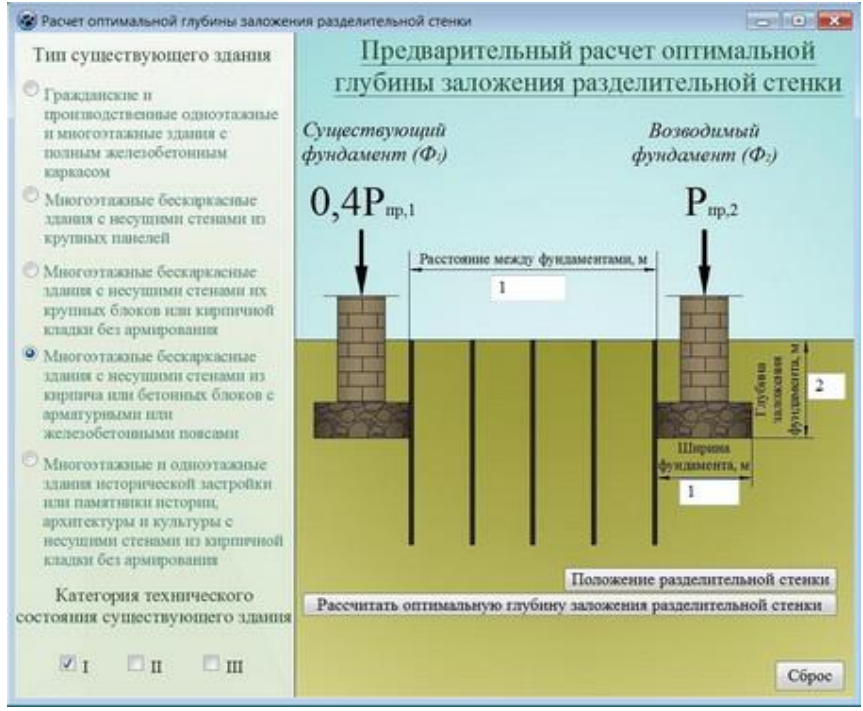

Рис. 8. Ввод исходных данных в программе «Расчет оптимальной глубины заложения разделительной стенки»

7) нажатием на кнопку «Положение разделительной стенки» активировать пять положений разделительной стенки для выбора, при этом цифра, указанная для каждого положения, определяет расстояние разделительной стенки от фундамента существующего строения;

8) выбрать проектное положение разделительной стенки (или близкое к проектному) (рис. 9);

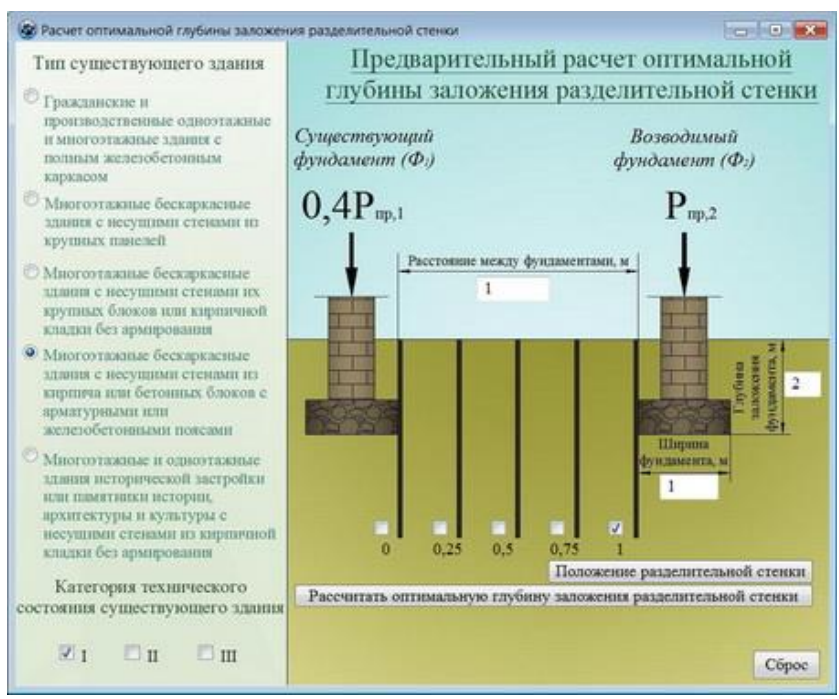

Рис. 9. Выбор положения разделительной стенки в программе «Расчет оптимальной глубины заложения разделительной стенки» 
9) расчет выполняется нажатием на кнопку «Рассчитать оптимальную глубину заложения разделительной стенки». Программа выдает расчетное значение глубины заложения разделительной стенки, а также нормативное и прогнозируемое значение отношения дополнительной осадки существующего здания к основной (рис. 10).

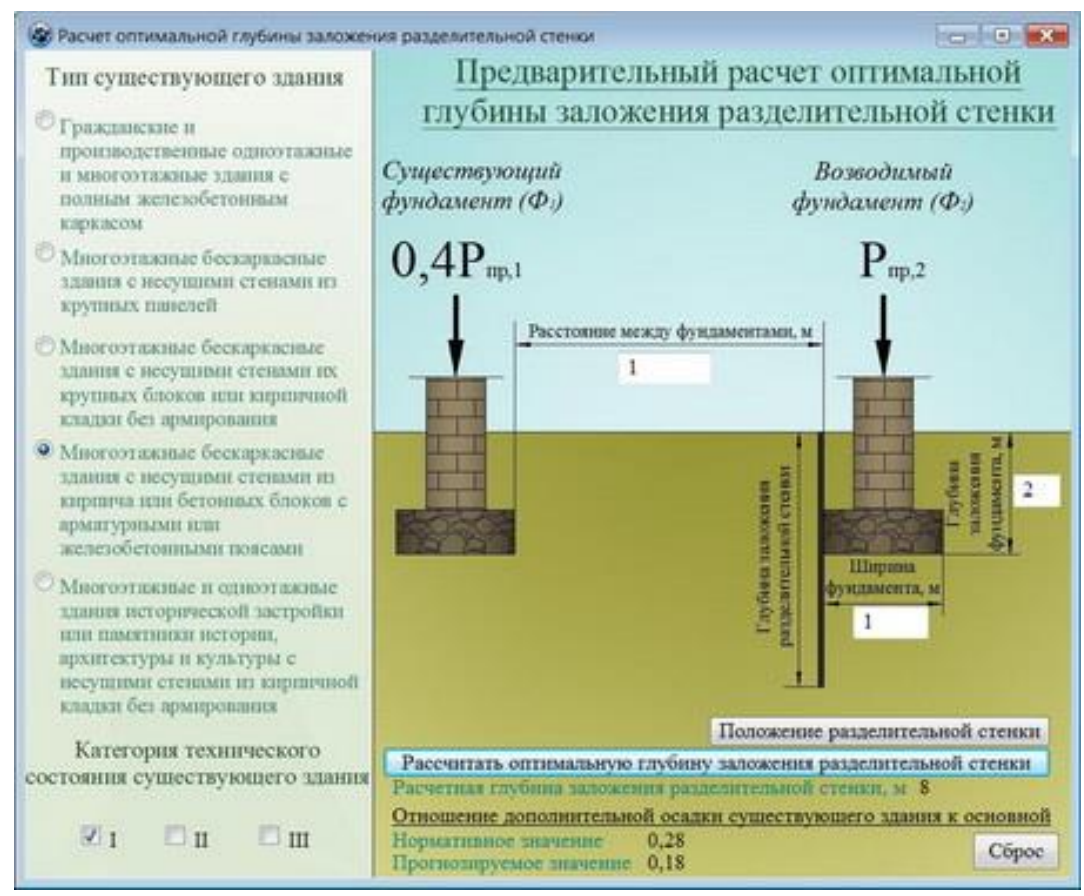

Рис. 10. Результат предварительного расчета оптимальной глубины заложения разделительной стенки

Таким образом, теоретически и эмпирически доказана эффективность разъединительного ряда как специального защитного мероприятия существующего строения от воздействий нового строительства. Получены новые данные изменения напряженно-деформированного состояния основания в зоне взаимовлияния соседних фундаментов, дана оценка эффективности разделительного шпунтового ряда для разных условий строительства. Разработана методика определения оптимальной глубины заложения разделительной стенки, реализованная в компьютерной программе, что значительно облегчит труд проектировщиков в определении параметров специальных защитных мероприятий по защите существующих строений от влияния нового строительства. 


\section{Библиографический список}

1. Чиж И.Н., Скибин Г.М. Экспериментальное моделирование взаимодействия соседних фундаментов, разделенных шпунтовым ограждением. // Известия вузов. Северо-Кавказский регион. Технические науки. - 2013. - №4. - С. 54-58.

2. Чиж И.Н. Экспериментально-численное исследование процесса взаимовлияния соседних фундаментов, разделенных шпунтовым рядом // Известия вузов. Северо-Кавказский регион. Технические науки. - 2013. - № 6. - С. 104-107.

3. Чиж И.Н. О влиянии разделительного ряда на напряженнодеформированное состояние оснований зданий в зоне нового строительства // Промышленное и гражданское строительство. - 2013. - № 11.

\section{References}

1. Chizh I.N., Skibin G.M. Jeksperimental'noe modelirovanie vzaimodejstviya sosednikh fundamentov, razdelennykh shpuntovym ograzhdeniem [Experimental simulation of the interaction of adjacent foundations separated by enclosing sheeting]. Izvestija vuzov. Severo-Kavkazskij region. Tekhnicheskie nauki, 2013. no. 4. pp. 54-58.

2. Chizh I.N. Jeksperimental'no-chislennoe issledovanie protsessa vzaimovliyaniya sosednikh fundamentov, razdelennykh shpuntovym ryadom [Experimental and numerical study of the process of mutual influence of neighboring bases, separated by enclosing sheeting]. Izvestija Vuzov. Severo-Kavkazskij region. Tekhnicheskie nauki, 2013. no. 6. pp. 104107.

3. Chizh I.N. O vliyanii razdelitel'nogo ryada na napryazhennodeformirovannoe sostoyanie osnovanij zdanij $\mathrm{v}$ zone novogo stroitel'stva [On the effect of separation on the number of stress-strain state of the bases of buildings in the area of new construction]. Promyshlennoe $i$ grazhdanskoe stroitel'stvo. 2013, no 11.

\section{Об авторах}

Чиж Ирина Николаевна (Новочеркасск, Россия) - аспирант Южно-Российского государственного политехнического университета (Новочеркасский политехнический институт) им. М.И. Платова; 
e-mail: dpchizh@mail.ru

Скибин Геннадий Михайлович (Новочеркасск, Россия) - доктор технических наук, профессор, декан строительного факультета, заведующий кафедрой «Промышленное и гражданское строительство, геотехника и фундаментостроение» Южно-Российского государственного политехнического университета (Новочеркасский политехнический институт) им. М.И. Платова; e-mail: skibingm@mail.ru

\section{About the authors}

Czyz Irina Nikolaevna (Novocherkassk, Russian Federation) - Doctoral Student, South-Russian State Technical University (Novocherkassk Polytechnic Institute) named. M.I. Platov; e-mail: dpchizh@mail.ru

Skibin Gennady Mikhailovich (Novocherkassk, Russian Federation) Doctor of Technical Sciences, Professor, Dean of Civil Engineering Faculty, Head of Department "Industrial and civil engineering, geotechnical and foundation", South-Russian State Technical University (Novocherkassk Polytechnic Institute) named. M.I. Platov; e-mail: skibingm@mail.ru

Получено 25.03.2014 